\title{
The Physical Foundation of Car-following Model
}

\author{
Xiaolin Shu, Shuo Jin, Ying Zhang, Hongbo Zhou, Guanghong Lu \\ College of Physical Science and Nuclear Engineer, Beihang University, Beijing, 100191, China \\ Email: talis.bachmann@ut.ee
}

Received 2012

\begin{abstract}
Based on the Newton's law of motion, the motion equation of the microscopic car-following model is introduced. The moving vehicles comply with the idea of Newton's equations of motion. But the interaction between vehicles is different from their traction. The inertia of the car is not its mass. We discuss the differences and present how to treat them. This article can deepen and widen the students' understanding of Newton's laws of motion. It can also provide the materials for university physical teaching. It is suitable for the students to exploratory study.
\end{abstract}

Keywords: Component; Newton’s Law of Motion; the Car Following Model; Inertia

\section{Introduction}

Mechanics study the interactions of objects as well as their motion under the interactions. The Newton's three laws of motion are the basis of mechanics. The motion states of a particle can be determined by the Newton motion equation. In middle school, mechanics is the main part of the physics teaching. The students have known very well about how to solve linear motion and uniform circular motion with Newton's law. In college physics teaching, the basic laws of mechanics, including Newton's three laws of motion, work-energy theorem and impulse-momentum theorem, have been teaching in classroom systematically. The particle dynamics problems can be divided into two types. (1) Knowing particles equation of motion, to find the action on it force. This kind of problem can be adopted by the differential method. (2) Knowing force on a particle, to find the equation of motion. The kind of problem can be worked out by integral method. A variety of cases of these two kinds of problems are teaching in classroom, such as the external force on particle is a function of time, or position or velocity, etc. It is also given out the solving steps for every cases. Through the accumulated effect of exerting a force on a body that undergoes a displacement, work-energy theorem is introduced. By the accumulation effect of the net force acting on the object during the interval, the impulse-momentum theorem is introduced. On the classroom teaching systematically and large exercise training after class, the students master the fixed steps and thinking mode on solving problems by Newton's law. In principle, students should be able to solve all the particle dynamics' problems.

But some factors becomes the obstacles to develop the ability of applying knowledge to solve actual problems. Such as the teaching materials are out of line with the actual living cases, and the teaching goal is for the entrance examination, etc. Even though students know well about the teaching physical content and have a large number of similar repeated training exercises, it is restrained the students' minds of students. This paper analyzes that the Newton's motion equation applied in traffic problem to deduce the microscopic car-follow model equation. On the one hand, to promote the students' interest in university physics, on the other hand, to display application of physical basic principles in the solution actual problem.

High efficient transport system is the development foundation of modern industrial society. With the rapid development of industry, the traffic volume increasing rapidly in many countries is over the capacity of the highway. It has seriously hindered the development of society. Understanding transportation process and the dynamics law is the basis of solving the traffic problems. As in 1935, Greenshields [1] started to study the vehicle traffic problems. Transportation process shows so many physical phenomena, such as dynamic jam, phase change, critical phenomenon, metastability, self-organized critical state, nonlinear wave (solitary wave), etc. These phenomena attracted the physicists' wide interests. They study the traffic problems from physics thoughts. It is said that the development of the modern traffic theory is the organic combination of the computer technology and modern physics technology. Helbing [2,3] carried on the detailed conclusion on traffic physical model. Microscopic car-following model is a good example for applying the classical Newton's motion equation to solve the traffic problem.

\section{Microscopic Car Following Model}

Newton's second law of motion describes the reason for the variation of the motion state of the particle. It is the relationship between the net force and the changes of motion state. It states as that the net force vector is equal to the mass $m_{\alpha}$ of the object times the acceleration of the object.

$$
m_{\alpha \alpha \beta} \frac{d^{2} \vec{r}_{\alpha}(t)}{d t^{2}}=\sum_{\beta(\alpha)} \vec{F}
$$

where $\vec{F}_{\alpha \beta}$ is the force between particles $\alpha$ and $\beta$, which is related with position or velocity of two particles.

Consider the traffic flow as a particle system and the simple case of linear movement as the friction $F_{f r}(t)=-\mu_{\alpha} v_{\alpha}(t)$ with friction coefficient $\mu_{\alpha}$, so the Newton motion equation of particle (1) can be expressed as:

$$
\left.m_{\alpha a x a d a \beta} \ddot{x}(t)=F_{0}(\alpha v(t), t) t\right)-\quad F \quad t \sum_{\beta(\alpha)} \quad()
$$

where $F_{0}\left(x_{\alpha}, t\right)$ is the vehicle driving force, which is the 
interaction between the particles. From this equation, it is deduced systematically to many physical quantities, such as the density of particles, momentum or average rate, etc.

Highway traffic can be regarded as the self-driving system. The moving car can be treated as particles in the system (as shown in Figure 1 below). Besides the external force, there are the internal forces from other particles in the system. The driving force of between cars can be used $F_{\alpha}^{0}(t)$ to instead $F_{0}\left(x_{\alpha}, t\right)$. The inertia is the property of the tendency of an object to keep moving once it is set in motion. Usually, the mass is a quantitative measure of inertia. In the traffic flow, the vehicle mass is not the inertia because it is the self-driving car. The inertia is related to the vehicle mass. But it is also related to the other factors, such as the driving force and braking force etc. So it is hard to decide. In the model, the quality $m_{\alpha}$ can be moved to the right side of the equation and combined with the force together as the force, which is also undetermined. This method is also applied to the calculation of density functional theory. Let $F_{\alpha a \alpha}^{0}(t) \neq \nu(t)^{p} \quad, \quad \gamma=\overline{\alpha a \alpha \alpha} m, \quad, \quad F_{\alpha \beta \alpha \beta \beta}(t)=m f(t)$, the equation (2) is as

$$
\frac{d v_{\alpha}(t) d}{d t \tau}=\frac{v^{0}(t) e^{0}(t)-v(t)}{\alpha}+\sum_{\beta(\alpha)} f_{\alpha \beta}(t)
$$

The meaning of the first term in the right of the equation is that in the relaxation time $\tau_{\alpha}$, the particle speed tends to the wanted velocity $v_{\alpha}^{0}(t)$ as the exponential function form on the effects of the driving force $v_{\alpha}^{0}(t) /$ and friction force $-v_{\alpha d}(t) /$. The $f_{\alpha \beta}(t)$ is the action from other particles. Obviously if the action is an attractive force with particle $\alpha$, it will lead to gather effect to form a blockage. So, it is usually use to reducing or exclusive action.

In 1950s, physicists Pipes put forward the microscopic traffic car-following model [4]. He assumed that the acceleration of vehicle $\alpha$ just determined by its front neighboring. Therefore, equation (3) reduces to:

$$
\frac{d v_{a(a)}(t)}{d t \tau}=\frac{v^{0}-v(t)}{a}+f_{a x a+1}(t)
$$

It should pay attention that the Newton's third law of motion is not suiting to in the case. The leading vehicle has an effect on its following vehicle, but the following vehicle has no action on its leading car.

The force $f_{\alpha, \alpha-1}(t)<0$, shows the repulsive action of vehicle $\alpha$ and its leading vehicle $(\alpha-1)$. It is influenced by three factors: (1). the relative speed $\Delta v_{\alpha}(t)$, (2). the safe distance $s_{\alpha}(t)$, (3). the speed $v_{\alpha}(t)$ of the vehicle $\alpha$. Forces $f_{\alpha, \alpha-1}(t)$ is the function of three amounts,

$$
f_{\alpha(\alpha)+a d}(t)=f(s 1(t) t t y)(t)
$$

when the vehicle is in the motion of constant acceleration, it can introduce the traffic correlation function of speed.

$$
v^{e}\left(s_{a, d u d a}\right)=v f\left(s_{0}, v y y\right)
$$

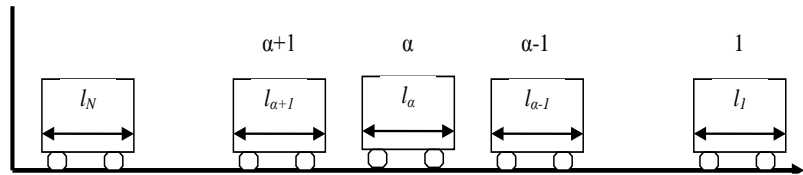

Figure 1.

Schematic plot for car flow [4].
Substitute (6) into (4), it can simplify model equation as

$$
\frac{d v_{\text {aqaak }}}{d t \tau} \frac{v^{e}\langle(s, \nu, v \quad-}{}
$$

Equation (7) is the basic equation microscopic car-following model.

Bando [5] supposes a specific form

$$
v_{e}^{\prime}(d)=\left(v_{0} / 2\right)\left[\tanh \left(d-d_{c}\right)+\tanh d_{c}\right]
$$

where $V_{0}$ the maximum speed of the vehicle, $d_{c}$ the safe distance. Using the optimal speed of traffic instead of the correlation function, the car-following model can be represent as

$$
\frac{d v_{a}(t)}{d t \tau}=\frac{v_{e}^{\prime}(d(t))-v(t)}{2}
$$

In Bando's simulation, the traffic appeared three different areas: stable area, metastability area and unstable region. When it is in stable condition $\frac{d v_{e}^{\prime}(d)}{d d_{a \alpha}}=\frac{d v(s)}{d s}>\frac{1}{2 \tau}$, which means the relaxation time is larger or the velocity is larger, only a tiny disturbance will cause traffic jams. Figure 2 is a system evolution plot of time and location structure by Bando [5]. In the picture, the black areas represent a higher density of vehicle which is easy to form traffic jams areas, gray area stand for the low density areas of vehicle. The high density areas is called jam phase, low density areas is called the movement phase.

\section{Conclusion}

From the above derivation process of microscopic traffic car-following model, the interaction between the vehicles is not the traction of cars. It is related to the safe distance of the car, the speed of the car and the speed difference from the leading car. The inertial is not the mass of vehicle because of the self-driving system. It can be treated as combining in the interaction. The Newton's third law is not suitable in that case. But the idea of Newton's motion law can still be used in the traffic problem because the motion and its state of vehicle are determined by the interaction.

In the microscopic car-following model established process, it is a very good example for how to treat and simplify the physical concept and the basic principles of physics. It is presented the practical application of the physical ideas and methods. This is the missing part of the classroom teaching. It is a 0

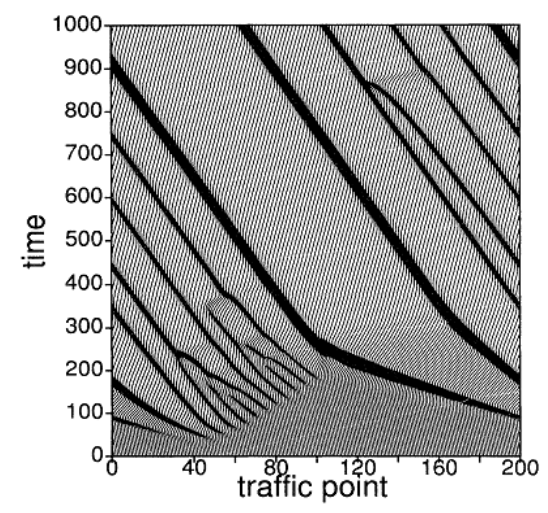

Figure 2. 
Plots of the positions of all vehicles [5].

important ability cultivation for students using learning knowledge to solve actual problem. This paper hope to provide materials for college physics teacher to teaching in class and for students to make creative learning

\section{REFERENCES}

B. D. Greenshields, “A Study of Traffic Capacity ,“ Proceedings of the highway research board (Highway Research Board, Washington, D.
C.), vol. 14, pp. 448-477, 1935.

Dirk Helbing, "Traffic and related self-driven many-particle systems," Review of Modern Physics, vol. 73, pp. 1067-1141, 2001.

T. Nagatani, “The physics of traffic jams,” Rep. Prog. Phys., vol. 65 pp. 1331-1386, 2002.

Louis A. Pipes, “An operational analysis of traffic dynamics,” Journal of Applied Physics, vol. 24, pp. 274-281, 1953.

M. Bando, K. Hasebe, A. Nakayama, A. Shibata, and Y. Sugiyama, "Dynamical model of traffic congestion and numerical simulation," Phys. Rev. E., vol. 51 pp. 1035-1042, 1995. 